\title{
Adaptive Distributed Space-Time Coding for Cooperative MIMO Relaying Systems with Limited Feedback
}

\author{
Tong Peng, Rodrigo C. de Lamare \\ Communications Research Group, \\ Department of Electronics, \\ University of York, \\ York YO10 5DD, UK \\ Email: tp525@ohm.york.ac.uk; rcd1500@ohm.york.ac.uk,
}

\author{
Anke Schmeink \\ TUMIC Research Centre, \\ RWTH Aachen University, \\ D-52056 Aachen, Germany \\ Email: schmeink@umic.rwth-aachen.de
}

\begin{abstract}
An adaptive distributed space-time coding (DSTC) scheme is proposed for two-hop cooperative MIMO networks. Linear minimum mean square error (MMSE) receive filters and adjustable matrices subject to a power constraint are considered with an amplify-and-forward (AF) cooperation strategy. In the proposed DSTC scheme, an adjustable matrix obtained by a feedback channel is employed to transform the space-time coded matrix at the relay node. Linear MMSE expressions of the adjustable code matrices based on the mean square error (MSE) and the maximum likelihood (ML) criteria are derived. The effects of the limited feedback and the feedback errors on the performance are considered. A stochastic gradient (SG) algorithm is also developed with reduced computational complexity. The simulation results show that the proposed algorithms obtain significant performance gains as compared to existing DSTC schemes.
\end{abstract}

\section{INTRODUCTION}

Cooperative multiple-input and multiple-output (MIMO) systems, which employ multiple relay nodes with antennas between the source node and the destination node as a distributed antenna array, apply distributed diversity gain and provide copies of the transmitted signals to improve the reliability of wireless communication systems [1]. Among the links between the relay nodes and the destination node, cooperation strategies, such as Amplify-and-Forward (AF), Decode-andForward (DF), and Compress-and-Forward (CF) [2] and relay selection algorithm [3] and various distributed space-time coding (DSTC) schemes in [4], [5] and [16] can be employed.

By employing a DSTC at the relay node in a cooperative network, providing more copies of the desired symbols at the destination node, the system can increase its diversity and coding gains to combat the interference. The recent focus on the DSTC technique lies in the design of delay-tolerant codes and full-diversity schemes with minimum outage probability. An opportunistic DSTC scheme with the minimum outage probability is designed for a DF cooperative network and compared with the fixed DSTC schemes in [6]. An adaptive distributed-Alamouti (D-Alamouti) STBC design is proposed in [7] for non-regenerative dual-hop wireless systems, which achieves the minimum outage probability.

The channel state information (CSI) is very important for a wireless communication system and can be estimated by sending a block of training symbols to the destination node. The feedback technique allows the destination node to transmit the CSI or other information back to the source node, in order to achieve gains by pre-processing the symbols. In [8], the trade-off between the length of the feedback symbols, which is related to the capacity loss, and the transmission rate is discussed, and in [9], one solution for this trade-off problem is derived. The use of limited feedback for STC encoding has been widely discussed in the literature. In [10], the phase information is sent back for STC encoding in order to maintain the full diversity, and the phase feedback is employed in [11] to improve the performance of the Alamouti STBC. The limited feedback is used in [12], [13] and [14] to provide the channel information for the pre-coding of an OSTBC scheme and blind adaptive estimation.

In this paper, we propose an adaptive distributed spacetime coding scheme based on the MSE and ML criteria for cooperative MIMO relaying systems with limited feedback. We focus on how the adjustable code matrix affects the DSTC during the encoding and how to optimize the linear receive filter with the adaptive matrix iteratively by the MSE criterion. The adjustable code matrices can be determined based on the ML criterion without the iteration but at the cost of a high computational complexity. It is shown that the utilization of an adjustable code matrix benefits the performance of the system compared to using traditional STC schemes. Then adaptive optimization algorithms are derived based on the MSE and ML criteria subject to constraints on the transmitted power at the relays, with the aid of a SG algorithm for the MSE criterion in order to release the destination node from the high computing complexity of the optimization process. The updated adjustable matrix is transmitted to the relay node through a feedback channel with errors, and the influence of the imperfect feedback is discussed.

The paper is organized as follows. Section II introduces a two-hop cooperative MIMO system with multiple relays applying the AF strategy and the randomized-DSTC (RSTC) scheme. In Section III the proposed optimization algorithm for the adaptive matrix is derived, and the results of the simulations are given in Section IV and Section V leads to the conclusion.

\section{Cooperative System Model}

The communication system under consideration, shown in Fig.1, is a cooperative communication system employing multi-antenna relay nodes transmitting through a MIMO channel from the source node to the destination node with feedback channels to the relay nodes. The 4-QAM modulation scheme is used in our system to generate the transmitted symbol vector $s[i]$ at the source node. There are $n_{r}$ relay nodes with $N$ antennas for transmitting and receiving, applying an AF cooperative strategy as well as a DSTC scheme, between the source node and the destination node. A two-hop communication system that broadcasts symbols from the source to $n_{r}$ relay nodes as well as to the destination node in the first phase, followed by 


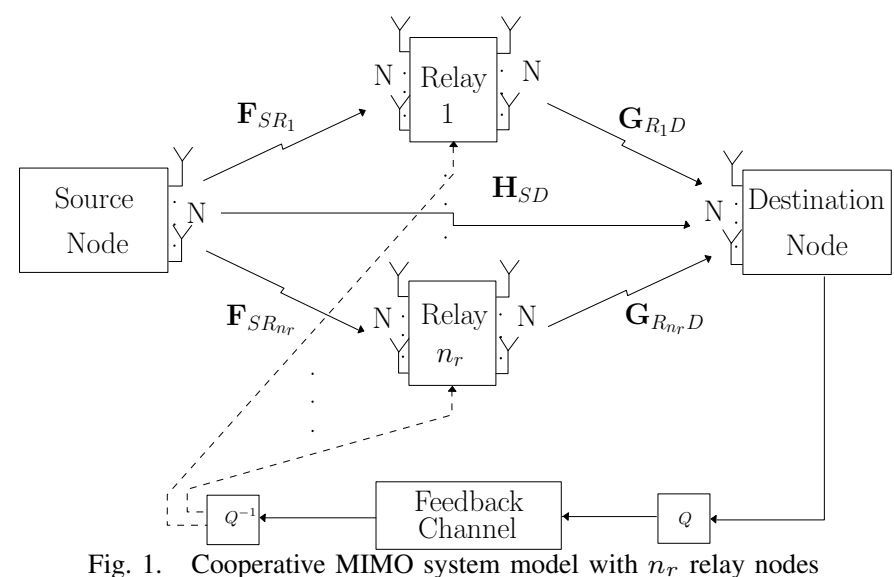

transmitting the amplified and re-encoded symbols from each relay node to the destination node in the next phase. After decoding at the destination node, the information matrix for encoding will be quantized first, and then transmitted back to each relay node through a feedback channel with noise and interference. The relay nodes quantize the feedback symbols and use them as a part of the encoding matrix in the next transmission. We consider only one user at the source node in our system that has $N$ Spatial Multiplexing (SM)-organized data symbols contained in each packet. The received symbols at the $k-t h$ relay node and the destination node are denoted as $\boldsymbol{r}_{S R_{k}}$ and $\boldsymbol{r}_{S D}$, respectively, where $k=1,2, \ldots, n_{r}$. The received symbols $\boldsymbol{r}_{S R_{k}}$ will be amplified before mapped into an STC matrix. We assume that the synchronization at each node is perfect. The received symbols at the destination node and each relay node can be described as follows

$$
\begin{gathered}
\boldsymbol{r}_{S D}[i]=\boldsymbol{H}_{S D}[i] \boldsymbol{s}[i]+\boldsymbol{n}_{S D}[i], \\
\boldsymbol{r}_{S R_{k}}[i]=\boldsymbol{F}_{S R_{k}}[i] \boldsymbol{s}[i]+\boldsymbol{n}_{S R_{k}}[i], \\
i=1,2, \ldots, N, \quad k=1,2, \ldots n_{r},
\end{gathered}
$$

where the $N \times 1$ vector $\boldsymbol{n}_{S R_{k}}[i]$ and $\boldsymbol{n}_{S D}[i]$ denote the zero mean complex circular symmetric additive white Gaussian noise (AWGN) vector generated at each relay and the destination node with variance $\sigma^{2}$. The transmitted symbol vector $\boldsymbol{s}[i]=\left[s_{1}[i], s_{2}[i], \ldots, s_{N}[i]\right]$ contains $N$ parameters, and has a covariance matrix $E\left[s[i] s^{H}[i]\right]=\sigma_{s}^{2} \boldsymbol{I}$, where $E[\cdot]$ stands for expected value, $(\cdot)^{H}$ denotes the Hermitian operator, $\sigma_{s}^{2}$ is the signal power which we assume to be equal to 1 and $\boldsymbol{I}$ is the identity matrix. $\boldsymbol{F}_{S R_{k}}[i]$ and $\boldsymbol{H}_{S D}[i]$ are the $N \times N$ channel gain matrices between the source node and the $k-t h$ relay node, and between the source node and the destination node, respectively.

After processing and amplifying the received vector $\boldsymbol{r}_{S R_{k}}[i]$ at the $k-t h$ relay node, the signal vector $\tilde{\boldsymbol{s}}_{S R_{k}}[i]=$ $\boldsymbol{A}_{R_{k} D}[i]\left(\boldsymbol{F}_{S R_{k}}[i] \boldsymbol{s}[i]+\boldsymbol{n}_{S R_{k}}[i]\right)$ can be obtained and will be forwarded to the destination node. The amplified symbols in $\tilde{\boldsymbol{s}}_{S R_{k}}[i]$ will be re-encoded by an $N \times T$ DSTC scheme $\boldsymbol{M}(\tilde{\boldsymbol{s}}[i])$ and then multiplied by an $N \times N$ randomized matrix $\boldsymbol{\Phi}[i]$ in [18], then forwarded to the destination node. The relationship between the $k-t h$ relay and the destination node can be described as

$$
\begin{gathered}
\boldsymbol{R}_{R_{k} D}[i]=\boldsymbol{G}_{R_{k} D}[i] \boldsymbol{\Phi}[i] \boldsymbol{M}_{R_{k} D}[i]+\boldsymbol{N}_{R_{k} D}[i], \\
k=1,2, \ldots, n_{r},
\end{gathered}
$$

where the $N \times T$ matrix $M_{R_{k} D}[i]$ is the DSTC matrix employed at the relay nodes whose elements are the amplified symbols in $\tilde{\boldsymbol{s}}_{S R_{k}}[i]$. The $N \times T$ received symbol matrix $\boldsymbol{R}_{R_{k} D}[i]$ in (3) can be written as an $N T \times 1$ vector $\boldsymbol{r}_{R_{k} D}[i]$ given by

$$
\boldsymbol{r}_{R_{k} D}[i]=\sum_{j=1}^{N} \boldsymbol{\Phi}_{e q_{k_{j}}}[i] \boldsymbol{G}_{e_{q_{j}}}[i] \tilde{\boldsymbol{s}}_{S R_{k_{j}}}[i]+\boldsymbol{n}_{R_{k} D}[i],
$$

where the $N T \times N$ matrix $\boldsymbol{G}_{e q_{k}}[i]$ stands for the equivalent channel matrix which is the DSTC scheme $\boldsymbol{M}(\tilde{\boldsymbol{s}}[i])$ combined with the channel matrix $\boldsymbol{G}_{R_{k} D}[i]$ and the block diagonal $N T \times N T$ matrix $\boldsymbol{\Phi}_{e q_{k_{j}}}[i]$ denotes the equivalent adjustable matrix assigned for the $j-t h$ forwarded symbol at the relay node. The $N T \times 1$ equivalent noise vector $\boldsymbol{n}_{R_{k} D}[i]$ generated at the destination node contains the noise parameters in $\boldsymbol{N}_{R_{k} D}[i]$. After rewriting $\boldsymbol{R}_{R_{k} D}[i]$ we can consider the received symbol vector at the destination node as an $N\left(n_{r}+1\right)$ vector with two parts, one is from the source node and another one is the superposition of the received vectors from each relay node. Therefore, the received symbol vector for the cooperative MIMO network considered can be written as

$$
\begin{aligned}
\boldsymbol{r}[i]= & {\left[\begin{array}{l}
\boldsymbol{H}_{S D}[i] \boldsymbol{s}[i] \\
\sum_{k=1}^{n_{r}} \sum_{j=1}^{N} \boldsymbol{\Phi}_{e q_{k j}}[i] \boldsymbol{G}_{e q_{k j}}[i] \tilde{s}_{S R_{k j}}[i]
\end{array}\right] } \\
& +\left[\begin{array}{c}
\boldsymbol{n}_{S D}[i] \\
\boldsymbol{n}_{R D}[i]
\end{array}\right] \\
= & \sum_{j=1}^{N} \boldsymbol{D}_{D_{j}}[i] \tilde{\boldsymbol{s}}_{D_{j}}[i]+\boldsymbol{n}_{D}[i],
\end{aligned}
$$

where the $(T+1) N \times\left(n_{r}+1\right) N$ block diagonal matrix $\boldsymbol{D}_{D_{j}}[i]$ contains the $N \times N$ channel coefficients matrix $\boldsymbol{H}_{S D}[i]$ between the source node and the destination node, and the $N T \times N$ equivalent channel matrix $\boldsymbol{G}_{e q_{k}}[i]$ for $k=1,2, \ldots, n_{r}$ between each relay node and the destination node. We can consider it as the end-to-end channel gain matrix for the $j-t h$ symbol in $\tilde{\boldsymbol{s}}_{D_{j}}[i]$. The $\left(n_{r}+1\right) N \times 1$ noise vector $\boldsymbol{n}_{D}[i]$ contains the received noise vector at the destination node and the amplified noise vectors from each relay node, which can be derived as an AWGN vector with zero mean and covariance matrix $\sigma^{2}\left(1+\left\|\sum_{k=1}^{n_{r}} \boldsymbol{\Phi}_{e q_{k}}[i] \boldsymbol{G}_{e q_{k}}[i] \boldsymbol{A}_{R_{k} D}[i]\right\|_{F}^{2}\right) \boldsymbol{I}$, where $\|\boldsymbol{X}\|_{F}=\sqrt{\operatorname{Tr}\left(\boldsymbol{X}^{H} \cdot \boldsymbol{X}\right)}=\sqrt{\operatorname{Tr}\left(\boldsymbol{X} \cdot \boldsymbol{X}^{H}\right)}$ stands for the Frobenius norm.

\section{JOINT CONSTRAINED AdAPTIVE DSTC OPTIMIZATION AND LINEAR MMSE RECEIVER DESIGN}

As derived in the previous section, the DSTC scheme used at the relay node will be multiplied by an adjustable matrix subject to a power constraint before being forwarded to the destination node. In this section, we present the joint linear receiver and adjustable code matrix design and devise a constrained adaptive optimization algorithm based on an SG estimation algorithm [17] for determining the optimal adjustable matrix and the linear MMSE receive filters, and the adaptive optimization algorithm based on the ML criterion.

\section{A. Linear MMSE Receiver Design with Adaptive DSTC Opti- mization}

The linear MMSE receiver design and the optimal adaptive DSTC matrices subject to a transmit power constraint at the 
relays are derived as follows. By defining the $(T+1) N \times 1$ linear receive filter $\boldsymbol{w}_{j}$ to estimate the $j-t h$ symbol $s_{j}[i]$ and considering the received vector in (5), we propose the MSE based optimization with a power constraint at the destination node as

$$
\begin{array}{r}
{\left[\boldsymbol{w}_{j}, \boldsymbol{\Phi}_{e q_{k_{j}}}\right]=\arg \min _{\boldsymbol{w}_{j}, \boldsymbol{\Phi}_{e q_{j}}} E\left[\left\|s_{j}[i]-\boldsymbol{w}_{j}^{H} \boldsymbol{r}[i]\right\|^{2}\right],} \\
\text { subject to } \sum_{j=1}^{N} \operatorname{trace}\left(\boldsymbol{\Phi}_{\mathrm{eq}_{\mathrm{k}_{\mathrm{j}}}} \boldsymbol{\Phi}_{\mathrm{eq}_{\mathrm{k}_{\mathrm{j}}}}^{\mathrm{H}}\right) \leq \mathrm{P}_{\mathrm{R}},
\end{array}
$$

where $\boldsymbol{r}[i]$ denotes the received symbol vector at the destination node which contains the adaptive matrix with the power constraint $P_{R}$. If we only consider the received symbols from the relay node, the received symbol vector at the destination node can be expressed as

$$
\begin{aligned}
\boldsymbol{r}[i] & =\sum_{k=1}^{n_{r}} \sum_{j=1}^{N} \boldsymbol{\Phi}_{e q_{k_{j}}} \boldsymbol{G}_{e q_{k_{j}}}[i] \tilde{\boldsymbol{s}}_{S R_{k_{j}}}[i]+\boldsymbol{n}_{D}[i] \\
& =\sum_{k=1}^{n_{r}} \sum_{j=1}^{N} \boldsymbol{\Phi}_{e q_{k_{j}}} \boldsymbol{c}_{k_{j}}[i] s_{j}[i]+\boldsymbol{n}_{D_{e q}}[i],
\end{aligned}
$$

where $c_{k_{j}}[i]$ is an $N T \times 1$ matrix that contains all the complex channel gains and the amplified matrix assigned to the received symbol $s_{j}[i]$ at the relay node, and the noise vector $\boldsymbol{n}_{D_{e q}}[i]$ is a Gaussian noise vector with zero mean and variance $\sigma^{2}(1+\|$ $\left.\sum_{k=1}^{n_{r}} \boldsymbol{\Phi}_{e q_{k_{j}}} \boldsymbol{G}_{e q_{k_{j}}}[i] \boldsymbol{A}_{j}[i] \|_{F}^{2}\right)$. Therefore, we can rewrite the MSE cost function as in (7), and define the Lagrangian of the constrained MSE minimization problem in (7) as

$$
\begin{aligned}
\mathscr{L}_{\boldsymbol{w}_{j}, \mathbf{\Phi}_{e q_{k j}}}= & E\left[\left\|s_{j}[i]-\boldsymbol{w}_{j}^{H} \boldsymbol{r}[i]\right\|^{2}\right] \\
& +\lambda\left(\sum_{j=1}^{N} \operatorname{trace}\left(\boldsymbol{\Phi}_{\mathrm{eq}_{\mathrm{k} j}} \boldsymbol{\Phi}_{\mathrm{eq}_{\mathrm{k}_{\mathrm{j}}}^{\mathrm{H}}}\right)-\mathrm{P}_{\mathrm{R}}\right),
\end{aligned}
$$

where $\lambda$ denotes the Lagrange multiplier and needs to be adjusted before the transmission.

Since $\boldsymbol{w}_{j}$ can be optimized by expanding the righthand side of (7) and taking the gradient with respect to $\boldsymbol{w}_{j}^{*}$ and equating the terms to zero, we can obtain the $j-t h$ MMSE receive filter

$$
\boldsymbol{w}_{j}=\boldsymbol{R}^{-1} \boldsymbol{p},
$$

where $\boldsymbol{R}=E\left[\boldsymbol{r}[i] \boldsymbol{r}^{H}[i]\right]$ denotes the auto-correlation matrix and $\boldsymbol{p}=E\left[\boldsymbol{r}[i] s_{j}^{*}[i]\right]$ stands for the cross-correlation vector. By optimizing the adjustable matrix $\boldsymbol{\Phi}_{e q_{k j}}$ for each symbol at each relay node, we can take the gradient of (8) with respect to $\boldsymbol{\Phi}_{e q_{k j}}^{*}$ and equate the terms to zero, resulting in

$$
\boldsymbol{\Phi}_{e q_{k_{j}}}=\tilde{\boldsymbol{R}}^{-1} \tilde{\boldsymbol{P}}
$$

where $\tilde{\boldsymbol{R}}=E\left[\left|s_{j}[i]\right|^{2} \boldsymbol{w}_{j} \boldsymbol{w}_{j}^{H}+\lambda \boldsymbol{I}\right]$ and $\tilde{\boldsymbol{P}}=$ $E\left[\left|s_{j}[i]\right|^{2} \boldsymbol{w}_{j} \boldsymbol{c}_{k_{j}}^{H}[i]\right]$. The power constraint can be enforced by multiplying the quotient of $P_{R}$ and the trace of the updated adjustable matrix. The expression in (10) does not provide a closed-form solution of the adjustable matrix $\boldsymbol{\Phi}_{e q_{k}}$ assigned for the $j-t h$ received symbol at the $k-t h$ relay node because it requires the adjustment of $\lambda$. This parameter needs to be adjusted in order to enforce the power constraint. Moreover, the expression in (10) also requires an inversion calculation with a high computational complexity. With the increase of the number of antennas employed at each node or employing more complicated STC encoders at the relay nodes, the complexity increases exponentially according to the matrix size in (10).

\section{B. Adaptive Matrix Optimization Algorithm with Linear MMSE Receiver Design}

In order to reduce the computational complexity of the proposed design and compute the required parameters, an adaptive robust matrix optimization (ARMO) algorithm with linear receiver design is proposed. We resort to a strategy that initially drops the power constraint, obtain the necessary recursions and then enforce the constraint with a normalization step. A simple adaptive algorithm for determining the linear receive filters and the adjustable matrices can be achieved by taking the instantaneous gradient terms of (8) with respect to $\boldsymbol{w}_{j}^{*}[i]$ and with respect to $\boldsymbol{\Phi}_{e q_{k j}}^{*}[i]$, respectively, which are

$$
\begin{aligned}
\nabla \mathscr{L}_{\boldsymbol{w}_{j}^{*}[i]} & =\nabla E\left[\left\|s_{j}[i]-\boldsymbol{w}_{j}^{H}[i] \boldsymbol{r}[i]\right\|^{2}\right]_{\boldsymbol{w}_{j}^{*}[i]} \\
& =-\left(s_{j}[i]-\boldsymbol{w}_{j}^{H}[i] \boldsymbol{r}[i]\right)^{H} \boldsymbol{r}[i]=-e_{j}^{*}[i] \boldsymbol{r}[i], \\
\nabla \mathscr{L}_{\boldsymbol{\Phi}_{e q_{k j}}^{*}[i]} & =\nabla E\left[\left\|s_{j}[i]-\boldsymbol{w}_{j}^{H}[i] \boldsymbol{r}[i]\right\|^{2}\right]_{\boldsymbol{\Phi}_{e q_{k j}}^{*}[i]} \\
& =-e_{j}[i] s_{j}^{*}[i] \boldsymbol{w}_{j}[i] \boldsymbol{c}_{k_{j}}^{H}[i],
\end{aligned}
$$

where $e_{j}[i]$ stands for the $j-t h$ detected error. After we obtain (11) the proposed algorithm is obtained by introducing a step size into the recursions. The proposed algorithm is given by

$$
\begin{aligned}
\boldsymbol{w}_{j}[i+1] & =\boldsymbol{w}_{j}[i]+\beta\left(e_{j}^{*}[i] \boldsymbol{r}[i]\right), \\
\boldsymbol{\Phi}_{e q_{k_{j}}}[i+1] & =\boldsymbol{\Phi}_{e q_{k_{j}}}[i]+\mu\left(e_{j}[i] s_{j}^{H}[i] \boldsymbol{w}_{j}[i] \boldsymbol{c}_{k_{j}}^{H}[i]\right), \\
\boldsymbol{\Phi}_{e q_{k_{j}}}[i+1] & =\frac{\sqrt{\mathrm{P}_{\mathrm{R}}} \boldsymbol{\Phi}_{e q_{k_{j}}}[i+1]}{\sqrt{\sum_{j=1}^{N} \operatorname{trace}\left(\boldsymbol{\Phi}_{\mathrm{eq}_{\mathrm{k}}}[\mathrm{i}+1] \boldsymbol{\Phi}_{\mathrm{eq}_{\mathrm{k}_{\mathrm{j}}}}^{\mathrm{H}}[\mathrm{i}+1]\right)}},
\end{aligned}
$$

where $\beta$ and $\mu$ denote the step sizes for the recursions for the estimation of the linear MMSE receive filter and the adaptive matrix for the DSTC scheme, respectively. The last equation in (12) stands for the normalization of the adaptive matrix after the iteration. According to (12), the receive filter and the matrix depend on each other, so that the algorithm in [14] can be used to determine the linear MMSE receive filter and the adaptive matrix iteratively, and the design can be achieved. The complexity for calculating the optimal $\boldsymbol{w}_{j}[i]$ and $\boldsymbol{\Phi}_{e q_{k_{j}}}[i]$ is $\mathrm{O}(N T)$ and $\mathrm{O}\left(N^{2} T^{2}\right)$, respectively, which is much less than $O\left(2 N^{3} T^{3}\right)$ and $O\left(2 N^{4} T^{4}\right)$ by using (9) and (10). As mentioned in Section I, the adaptive matrix will be sent back to the relay nodes via a feedback channel which requires quantization as will be shown in the simulations.

\section{Adaptive Matrix Optimization Algorithm based on $M L$ Criterion}

The ARMO algorithm is flexible and can use different criteria for optimization of the adjustable code matrix. In this section we will introduce an ARMO algorithm based on the maximum likelihood (ML) criterion. We also employ an ML detector for the algorithm, which provides the optimal performance at the cost of a high computational complexity. We have to store an $N \times D$ matrix $S$ at the destination node which contains all the possible combinations of the transmitted 


$$
\left[\boldsymbol{w}_{j}, \boldsymbol{\Phi}_{e q_{k_{j}}}\right]=\arg \min _{\boldsymbol{w}_{j}, \mathbf{\Phi}_{e q_{k_{j}}}} E\left[\left\|s_{j}[i]-\boldsymbol{w}_{j}^{H}\left(\sum_{k=1}^{n_{r}} \sum_{j=1}^{N} \boldsymbol{\Phi}_{e q_{k_{j}}} \boldsymbol{c}_{k_{j}}[i] s_{j}[i]+\boldsymbol{n}_{D}[i]\right)\right\|^{2}\right], \quad \text { s.t. } \quad \sum_{j=1}^{N} \operatorname{trace}\left(\boldsymbol{\Phi}_{\mathrm{eq}_{\mathbf{k}_{\mathbf{j}}}} \boldsymbol{\Phi}_{\mathrm{eq}_{\mathbf{k}_{\mathrm{j}}}}^{\mathrm{H}}\right) \leq \mathrm{P}_{\mathrm{R}} .
$$

symbol vectors for an ML detector. The ML optimization problem can be written as

$$
\begin{aligned}
& {\left[\hat{s}_{d_{j}}[i], \hat{\boldsymbol{\Phi}}_{e q_{k_{j}}}[i]\right]=} \\
& \arg \min _{s_{d_{j}}[i], \boldsymbol{\Phi}_{e q_{k_{j}}}[i]}\left\|\boldsymbol{r}[i]-\left(\sum_{k=1}^{n_{r}} \sum_{j=1}^{N} \boldsymbol{\Phi}_{e q_{k_{j}}}[i] \boldsymbol{c}_{k_{j}}[i] s_{d_{j}}[i]\right)\right\|^{2}, \\
& \text { for } d=1,2, \ldots, D,
\end{aligned}
$$

where $s_{d_{j}}[i]$ stands for the $(j, d)$ th element in the symbol matrix $\boldsymbol{S}$. By substituting each column of $\boldsymbol{S}$ into (13), we can obtain the most likely transmitted symbol vector $\hat{\boldsymbol{s}}_{d}[i]$. After determining the transmitted symbol vector $\hat{\boldsymbol{s}}_{d}[i]$, we can calculate the optimal code matrix $\boldsymbol{\Phi}_{e q_{k}}[i]$. Define $\hat{\boldsymbol{r}}[i]=$ $\sum_{k=1}^{n_{r}} \sum_{j=1}^{N} \boldsymbol{\Phi}_{e q_{k_{j}}}[i] \boldsymbol{c}_{k_{j}}[i] \hat{s}_{d_{j}}[i]$ which stands for the received symbol vector without noise. By expanding the righthand side of (13) we obtain

$$
\begin{aligned}
\mathscr{L} & =\|\boldsymbol{r}[i]-\hat{\boldsymbol{r}}[i]\|^{2} \\
& =(\boldsymbol{r}[i]-\hat{\boldsymbol{r}}[i])^{H}\left(\boldsymbol{r}[i]-\sum_{k=1}^{n_{r}} \sum_{j=1}^{N} \boldsymbol{\Phi}_{e q_{k_{j}}}[i] \boldsymbol{c}_{k_{j}}[i] \hat{s}_{d_{j}}[i]\right) .
\end{aligned}
$$

The ML optimization problem in (14) is equivalent to a leastsquares (LS) problem in this case, so we can solve the problem by taking the instantaneous gradient of (14) with respect to the code matrix $\boldsymbol{\Phi}_{e q_{k_{j}}}^{*}[i]$, which gives

$$
\nabla \mathscr{L}_{\boldsymbol{\Phi}_{e q_{k_{j}}}^{*}[i]}=-\hat{s}_{d_{j}}^{*}[i](\boldsymbol{r}[i]-\hat{\boldsymbol{r}}[i]) \boldsymbol{c}_{k_{j}}^{H}[i],
$$

and the computation of the optimal $\boldsymbol{\Phi}_{e q_{k_{j}}}[i]$ requires $\nabla \mathscr{L}_{\boldsymbol{\Phi}_{e q_{k j}}^{*}[i]}=0$, and by substituting $\hat{\boldsymbol{r}}[i]$ into (15) we can obtain the optimal adjustable code matrix as given by

$$
\boldsymbol{\Phi}_{e q_{k_{j}}}[i]=\hat{s}_{d_{j}}^{*}[i] \boldsymbol{r}_{e_{j}}[i] \boldsymbol{c}_{k_{j}}^{H}[i]\left(\left|\hat{s}_{d_{j}}[i]\right|^{2} \boldsymbol{c}_{k_{j}}[i] \boldsymbol{c}_{k_{j}}^{H}[i]\right)^{\dagger},
$$

where $(.)^{\dagger}$ stands for pseudo-inverse, and $\boldsymbol{r}_{e_{j}}[i]=\boldsymbol{r}[i]-$ $\sum_{k=1}^{n_{r}} \sum_{l=1, l \neq j}^{N} \boldsymbol{\Phi}_{e q_{k_{l}}}[i] \boldsymbol{c}_{k_{l}}[i] \hat{s}_{d_{l}}[i]$. The optimal code matrices will be normalized in order to eliminate the energy introduced during the optimization and then transmitted back to the relay nodes.

\section{Simulations}

The simulation results are shown here to assess the proposed scheme and algorithm. The system considered is an AF cooperative MIMO system with the Alamouti STBC scheme using QPSK modulation in quasi-static block fading channels with AWGN, as derived in Section II. The bit error rate (BER) performance of the proposed ARMO algorithm with the linear receiver design is assessed, and the influence of the imperfect feedback channels are considered in the simulations. The system employs $n_{r}=1$ relay node and each node in the system has $N=2$ antennas. In the simulation, we define both the symbol power at the source node to be equal to 1. The RSTC scheme is designed by multiplying the $2 \times$ 2 Alamouti STBC [15] by a randomized matrix with each

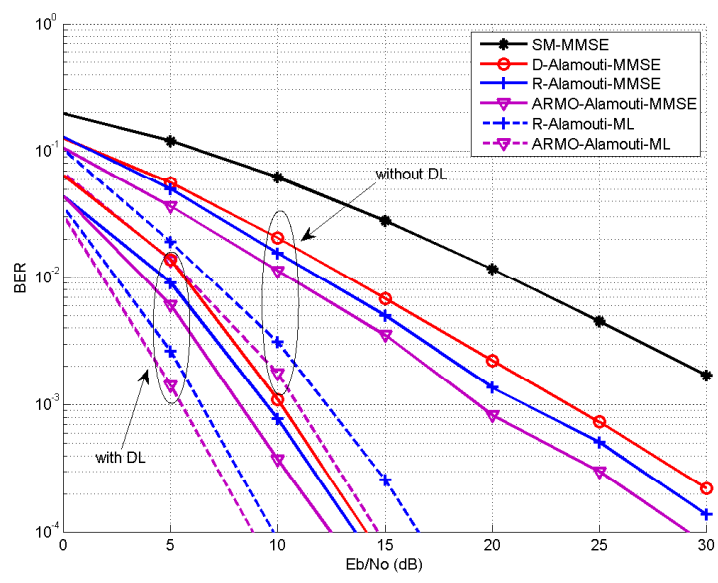

Fig. 2. BER performance vs. $E_{b} / N_{0}$ for ARMO Algorithm with and without the direct link

element generated using $e^{j \theta}$ where $\theta$ is uniformly distributed in $[0,2 \pi)$.

The proposed ARMO algorithm with different detection algorithms are compared with the SM scheme and the RSTC algorithm using the D-Alamouti STBC scheme in [16] with $n_{r}=1$ relay nodes in Fig. 2. The results illustrate that without the direct link, by making use of the STC or the RSTC technique, a significant performance improvement can be achieved compared to the spatial multiplexing system. The RSTC algorithm outperforms the STC-AF system, while the ARMO algorithm can improve the performance by about $3 \mathrm{~dB}$ as compared to the RSTC algorithm. With the consideration of the direct link, the results indicate that the cooperative diversity order can be increased, and using the ARMO algorithm achieves an improved performance with $2 \mathrm{~dB}$ of gain as compared to employing the RSTC algorithm and $3 \mathrm{~dB}$ of gain as compared to employing the traditional STC-AF algorithm. By making use of the ML detector, the curves of the R-Alamouti and the ARMO algorithm can achieve the full diversity order and obtain a significant improvement in terms of gains compared to that with an MMSE detector, and it is obvious that the ARMO algorithm outperforms the RAlamouti with or without the direct link.

The simulation results shown in Fig. 3 illustrate the impact of the feedback channel on the ARMO algorithm. As mentioned in Section I, the optimal adaptive matrix will be sent back to each relay node through a feedback channel. The quantization and feedback errors are not considered in the simulation results in Fig. 2, so the optimal adjustable matrix is perfectly known at the relay node after the ARMO algorithm converges; while in Fig. 3, it is indicated that the performance of the proposed algorithm will be affected by the accuracy of the feedback information. In the simulation, we use 4 bits to quantize the real part and the imaginary part of each element of the adaptive matrix $\boldsymbol{\Phi}_{e q_{k_{j}}}[i]$, and the feedback channel is modeled as a binary symmetric channel. As we can see from Fig. 3, by decreasing the error probabilities for the feedback channel with fixed quantization bits, the BER 


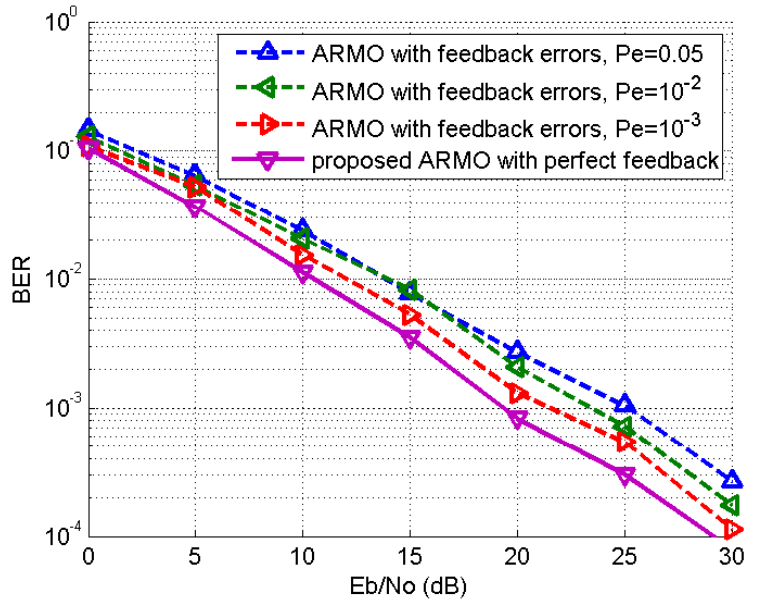

Fig. 3. BER performance for ARMO algorithm with perfect and imperfect feedback links with different $P_{e}$, quantization bits $=4$

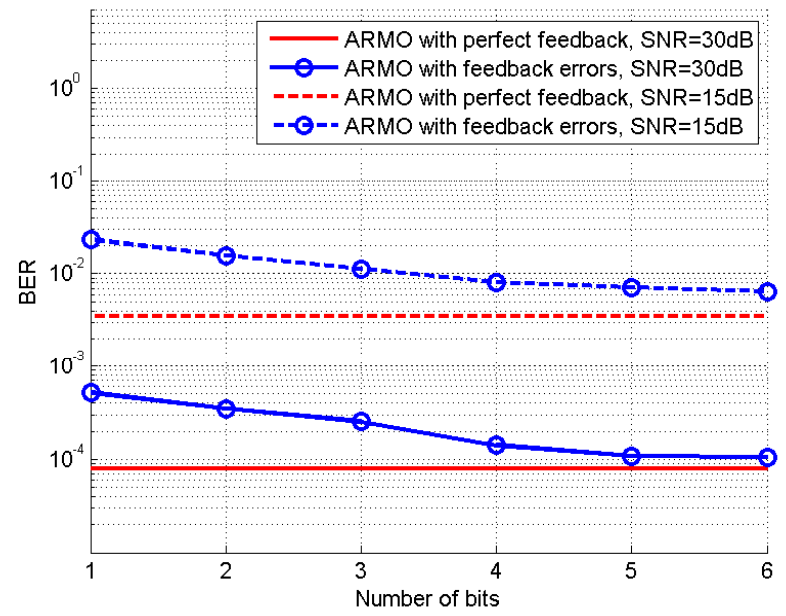

Fig. 4. BER performance vs. different number of bits for feedback quantization, $P_{e}=10^{-3}$

performance approaches the performance with the perfect feedback, and by making use of 4 quantization bits for the real and imaginary part of each parameter in the randomized matrix, the performance of the ARMO algorithm is about $1 \mathrm{~dB}$ worse with a feedback error probability of $10^{-3}$.

In Fig. 4, the influence of different quantization bits with fixed feedback error probabilities on the performance of the ARMO algorithm is evaluated. The BER performance with a perfect feedback channel is given as a lower bound with $S N R=15 d B$ and $S N R=30 d B$, respectively. The error probability is fixed in $P_{e}=10^{-3}$. It is obvious that with the increase of the number of bits employed in the quantization, the BER curves approach the result with perfect feedback due to a more accurate estimation, while the computational cost increases. In $S N R=30 d B$ circumstance, the result of 4-bit quantization approaches the result using a perfect feedback with a relatively low increase of computational complexity, and the BER loss is about $4 \times 10^{-4}$ compared to the result with perfect feedback.

\section{CONCLUSION}

We have proposed an adaptive robust matrix optimization (ARMO) algorithm with linear receiver filter design for the adaptive DSTC in a cooperative system. A joint iterative estimation algorithm for computing the receive filters and the adaptive matrix has been derived. The effect of the limited feedback and feedback errors have been considered in the simulations. The simulation results illustrate the advantage of the proposed ARMO algorithm by comparing it with the cooperative network employing the traditional DSTC scheme and the fixed RSTC scheme. The proposed algorithm can be used with different DSTC schemes using the AF strategy and can also be extended to the DF cooperation protocol.

\section{REFERENCES}

[1] P. Clarke, R. C. de Lamare, Joint Transmit Diversity Optimization and Relay Selection for Multi-relay Cooperative MIMO Systems Using Discrete Stochastic Algorithms, IEEE Communications Letters, vol. 15, p.p. 1035-1037, Oct. 2011.

[2] J. N. Laneman and G. W. Wornell, Cooperative Diversity in Wireless Networks: Efficient Protocols and Outage Behaviour, IEEE Transactons on Information Theory, vol. 50, no. 12, pp. 3062-3080, Dec. 2004.

[3] P. Clarke, R. C. de Lamare, Transmit Diversity and Relay Selection Algorithms for Multirelay Cooperative MIMO Systems, IEEE Transactions on Vehicular Technology, vol. 61, p.p. 1084-1098, March 2012.

[4] J. N. Laneman and G. W. Wornell, Distributed Space-Time-Coded Protocols for Exploiting Cooperative Diversity in Wireless Networks, IEEE Transsctions on Information Theory, vol. 49, no. 10, pp. 24152425, Oct. 2003.

[5] S. Yiu, R. Schober, L. Lampe, Distributed Space-Time Block Coding, IEEE Transactions on Wireless Communications, vol. 54, no. 7, pp 1195-1206, Jul. 2006

[6] Yulong Zou, Yu-Dong Yao, Baoyu Zheng, Opportunistic Distributed Space-Time Coding for Decode-and-Forward Cooperation Systems, IEEE Transactions on Signal Processing, vol. 60, pp. 1766 - 1781, April 2012.

[7] J. Abouei, H. Bagheri, A. Khandani, An Efficient Adaptive Distributed Space-Time Coding Scheme for Cooperative Relaying, IEEE Transactions on Wireless Communications, vol. 8, Issue: 10, pp. 4957-4962, October 2009.

[8] M. Kobayashi, G. Caire, N. Jindal, How Much Training and Feedback are Needed in MIMO Broadcast Channels?, IEEE International Symposium on Information Theory (ISIT), pp. 2663 - 2667, 6-11 July 2008 .

[9] A.D. Dabbagh, D.J. Love, Feedback Rate-Capacity Loss Tradeoff for Limited Feedback MIMO Systems, IEEE Transactions on Information Theory, vol. 52, p.p. 2190-2202, May 2006.

[10] J. Akhtar, D. Gesbert, Extending Orthogonal Block Codes with Partial Feedback, IEEE Transactions on Wireless Communications, vol. 3 , p.p. 1959-1962, Nov. 2004

[11] Choi Ilhwan, Kim Jong-Kyu, Lee Heunchul, Lee Inkyu, AlamoutiCodes Based Four-Antenna Transmission Schemes with Phase Feedback, IEEE Communications Letters, vol. 13, p.p. 749-751, Oct. 2009.

[12] G. Jongren, M. Skoglund, Quantized Feedback Information in Orthogonal Space-Time Block Coding, IEEE Transactions on Information Theory, vol. 50, p.p. 2473-2486, Oct. 2004.

[13] D.J. Love, R.W. Heath, Jr., Limited Feedback Unitary Precoding for Orthogonal Space-Time Block Codes, IEEE Transactions on Signal Processing, vol. 53, p.p. 64-73, Jan. 2005.

[14] R.C. de Lamare, R. Sampaio-Neto, Minimum Mean-Squared Error Iterative Successive Parallel Arbitrated Decision Feedback Detectors for DS-CDMA Systems, IEEE Transactions on Communications, vol. 56, p.p. 778-789, May 2008.

[15] T. Peng, R.C. de Lamare,Adaptive Distributed Space-Time Coding for Cooperative MIMO Relaying Systems, 2012 International Symposium on Wireless Communication Systems (ISWCS), p.p. 611-615, 28-31 Aug. 2012.

[16] R.C. de Lamare, R. Sampaio-Neto, Blind Adaptive MIMO Receivers for Space-Time Block-Coded DS-CDMA Systems in Multipath Channels Using the Constant Modulus Criterion, IEEE Transactions on Communications, vol. 58, no. 1, Jan. 2010.

[17] R.C. de Lamare, P.S.R. Diniz, Blind Adaptive Interference Suppression Based on Set-Membership Constrained Constant-Modulus Algorithms with Dynamic Bounds, IEEE Transactions on Signal Processing, 2012.

[18] B. Sirkeci-Mergen, A. Scaglione, Randomized Space-Time Coding for Distributed Cooperative Communication, IEEE Transactions on Signal Processing, vol. 55, no. 10, Oct. 2007. 